\title{
Assessing the effects of government incentives on the performance of SMEs in food manufacturing sector
}

\begin{abstract}
Aim/Purpose: The Malaysian government has given numerous incentives to small and medium enterprises (SMEs), including those in the food manufacturing sector, in an attempt to boost their performance. This study aims to assess the effects of these incentives, particularly financial and tax incentives, on the performance of SMEs in the Malaysian food manufacturing sector.
\end{abstract}

Background: Millions of Ringgit has been allocated for the development of SMEs by the Malaysian government. The findings of this study aim to assist the policymakers in improving the current policies in incentive give outs to enhance the effectiveness and reduce the number of SMEs that were forced to close down in less than five years of operation.

Methodology: The study was conducted using structure, conduct, and performance (SCP) paradigm on secondary data from 140 companies over a period of five years $(2013-2017)$. Correlation analysis was done to explore the relationship between each explanatory market variables included in the SCP paradigm.

Contribution: This study provides insights into the effect of different types of government incentives on the performance of SMEs in the Malaysian food manufacturing sector.

Findings: The study found that financial and tax incentives gave different effects on the performance of SMEs in the Malaysian food manufacturing sector during the study period. Financial incentive shows a weak positive significant correlation with advertising-to-sales ratio (ASR), return on assets (ROA) and market share (MS) ratio while showing negative significant correlation towards capital intensity (CAP). On the other hand, tax incentive shows a strong significant positive correlation with MS and weak significant positive correlation with CAP, ROA and return on sales (ROS). This shows that financial incentive strongly correlates with SMEs' performance, whereas tax incentive is associated with market structure and conduct of SMEs in the Malaysian food manufacturing sector.

Recommendations for Practitioners: Firstly, the government should consider providing extra assistance to SMEs in entering the sector as entry barriers for the sector is relatively high. Focus can be given in increasing financial incentives at a more competitive rate as it can reduce debt or increase the firm's equity or aid firms in acquiring assets, which are crucial for efficient and effective production of processed food. Allocation of tax incentives should be reviewed as it does not have a strong correlation with firms' performance.

Recommendation for Researchers: There are limitations to the number of SMEs included in this study. Hence, researchers are recommended to have direct contact with more firms to ensure more accurate data. 
Impact on Society: With more efficient and effective policies in the government's financial and tax incentives, more allocation can be channeled to other areas that have direct implications to the citizen. Additionally, with better policies, more jobs will be created in the market, and a highly competitive market will lead to a production of higher quality products that can be enjoyed by the consumers.

Future Research: This study has contributed to the SCP paradigm as it demonstrated the effects of government financial and tax incentives on the market structure, conduct, and performance of SMEs in the Malaysian food manufacturing sector. Future researches might focus on non-financial incentives given out by the government such as human resource development, training, industrial infrastructure and amenities, technology development and capabilities, technology transfer, and organizational innovation.

Keyword: Small medium enterprises; Structure-conduct-performance; Food manufacturing industry; Financial incentives; Tax incentives 